${ }^{1}$ Facultad de Ciencias de la Salud. Departamento de Fisioterapia. Universidad de Granada. España. ${ }^{2}$ Facultad de Ciencias de la Salud. Departamento de Enfermería. Universidad de Granada. España.

Los autores declaran no tener conflictos de interés.

La presente investigación no ha recibido ayudas específicas provenientes de agencias del sector público, sector comercial o entidades sin ánimo de lucro.

Recibido el 10 de octubre de 2019, aceptado el 22 de enero de 2021.

Correspondencia a: Marie Carmen Valenza. Facultad de Ciencias de la Salud. Universidad de Granada. Av. De la llustración, 60. 18016 Granada, España. valenza@ugr.es

\section{Prevalencia y repercusión del síndrome locomotor en el perfil clínico de la enfermedad pulmonar obstructiva crónica}

\author{
RAMÓN ROMERO FERNÁNDEZ ${ }^{1}$, ANDRÉS CALVACHE MATEOํㅜ, \\ ESTHER PRADOS ROMÁN ${ }^{1}$, MARÍA GRANADOS SANTIAGO², \\ ARACELI ORTIZ RUBIO ${ }^{1}$, MARIE CARMEN VALENZA ${ }^{1 *}$
}

\section{Prevalence and repercussions of the locomotor syndrome in patients with chronic obstructive pulmonary disease}

\begin{abstract}
Background: Patients with Chronic Obstructive Pulmonary Disease (COPD) suffer a progressive deterioration of functional status and a decrease in independence in activities of daily living. Locomotor Syndrome (SL) is the involvement of the musculoskeletal system due to the deterioration caused by age. Aim: In patients with COPD, to evaluate the prevalence in LS and assess its impact on functional status and quality of life. Material and Methods: Cross sectional assessment of 259 patients with COPD. LS was evaluated with the Geriatric Locomotive Function Scale (GFLS-25). Those with a score $<16$ were classified as having LS. Functional status was evaluated with dynamometry, Five Times Sit-to-Stand test, and the Modified Baecke Physical Activity Questionnaire. Functional impairment was measured with the London Chest Activity of Daily Living, and the quality of life was evaluated with EuroQol-5D. Results: LS was found in 139 patients (53\%). Activity levels and muscle strength were lower in these patients. Also, they had a higher frequency of functional impairment and a lower quality of life perception. Conclusions: LS in patients with COPD impacts their functional status and quality of life.
\end{abstract}

(Rev Med Chile 2021; 149: 378-384)

Key words: Functional Status; Physical Functional Performance; Pulmonary Disease, Chronic Obstructive.

\section{L} a Enfermedad Pulmonar Obstructiva Crónica (EPOC) es una enfermedad progresiva cida por una limitación crónica del rcialmente irreversible ${ }^{1}$. Los princis síntomas clínicos son la disnea, la fatiga y la tos ${ }^{2}$. Con una prevalencia de $9,1 \%$ en la población adulta cursa con períodos de estabilidad clínica intercalados con exacerbaciones de la sintomato$\operatorname{logí}^{3}$. Diversos estudios ${ }^{4,5}$ han mostrado que los pacientes con EPOC desarrollan una reducción de la movilidad conocida como Síndrome Locomotor
(SL); afectando al estado funcional y a la calidad de vida de estos pacientes ${ }^{6}$.

El SL conlleva alteraciones en el sistema músculo-esquelético en los pacientes con $\mathrm{EPOC}^{7,8}$. Esto produce un deterioro de la fuerza muscular', que incluye la pérdida de densidad mineral ósea, reducción de sección transversal muscular y disminución de los niveles de actividad ${ }^{10,11}$. Por tanto, las alteraciones músculo-esqueléticas junto con la sintomatología propia del EPOC producen una mayor pérdida de funcionalidad, incremen- 
tando el deterioro físico y psicológico de estos pacientes $^{12,13}$.

Un estudio realizado sobre pacientes con patología respiratoria mostró una alta prevalencia de SL, demostrando una disminución en la calidad de vida ${ }^{14}$. Sin embargo, son escasos los estudios realizados para evaluar la relación del SL, la calidad de vida y la funcionalidad en pacientes con EPOC.

Por ello, el objetivo de este estudio es evaluar la prevalencia del SL y valorar la repercusión que tiene en la funcionalidad y en la calidad de vida de los pacientes con EPOC.

\section{Material y Método}

\section{Diseño}

Se realizó un estudio observacional transversal desde 2012 hasta 2018 en pacientes con EPOC y en un grupo control emparejado por edad y sexo. Este estudio tuvo la aprobación del Comité de Ética de Investigación Biomedicina de Granada, así como la Declaración de Helsinki de experimentaciones en humanos revisada en 2013. Los pacientes participaron de forma voluntaria y tras ser informados de las características y del procedimiento del estudio y estar de acuerdo con los objetivos del estudio y el carácter confidencial de este, firmaron el consentimiento informado.

\section{Participantes}

Los pacientes incluidos en el estudio pertenecían al Servicio de Neumología del Hospital Universitario San Cecilio y del Hospital Universitarios Virgen de las Nieves de Granada (España).

Los criterios de inclusión fueron los siguientes: 1) Pacientes con EPOC en periodo estable; 2) con un estado moderado-severo (GOLD III y IV según la clasificación GOLD) ${ }^{15}, 3$ ) edad superior a 65 años, 4) querer participar en el estudio y firmar el consentimiento informado.

Se excluyeron a los individuos con: 1) Presencia de exacerbación en el último mes; 2) presencia de alteraciones cognitivas, neurológicas o psiquiátricas; 3 ) problemas músculo-esqueléticos y/u ortopédicos severos; 4) cáncer o fallo orgánico; 4) no firmar el consentimiento informado.

\section{Variables de medida}

Tras la firma del consentimiento informado se realizó la recogida de los datos descriptivos, sociodemográficos y antropométricos de forma individual. Para evaluar el grado de morbi-mortalidad se empleó el índice de comorbilidad de Charlson $^{16}$. Cuanto mayor es la puntuación, mayor comorbilidad y riesgo de mortalidad presenta el paciente. La medición de la fuerza espiratoria en el primer segundo $\left(\mathrm{FEV}_{1}\right)$ se evaluó mediante la curva flujo/volumen mediante un espirómetro (Spirobank II; MIR, Rome, Italy) con turbina desechable para evitar posibles infecciones y con una pinza nasal para evitar las pérdidas de aire ${ }^{17,18}$. La percepción de la disnea se midió mediante la escala de Borg modificada ${ }^{19}$.

Las variables principales evaluadas fueron el estado físico, el estado funcional y la calidad de vida.

El estado físico del paciente se valoró a través de la fuerza de prensión de la mano derecha, la capacidad al ejercicio y los niveles de actividad. La fuerza de presión es un índice objetivo de la integridad funcional de la extremidad superior ${ }^{20}$, que detecta la pérdida de la función muscular fisiológica ${ }^{21}$. Se evaluó con un dinamómetro de presión cuantificado en Newton siguiendo un protocolo validado ${ }^{22}$. La capacidad al ejercicio se midió mediante el Five-Time-Sit-to-Stand Test $(5 \mathrm{STS})^{23}$. Es una herramienta de miembros inferiores empleada para evaluar el tiempo que tardan los pacientes en levantarse y sentarse 5 veces de una silla sin emplear apoyos ${ }^{24}$. Finalmente, el cuestionario MBPAQ (Modified Baecke Physical Activity Questionnaire) evalúa el nivel de actividad física de forma específica en personas mayores de 60 años, expresando los valores más bajos un mayor sedentarismo ${ }^{25}$.

La disnea relacionada con el estado funcional se midió mediante el cuestionario London Chest Activity of Daily Living Scale (LCADL). El cuestionario consta de 15 ítems con 4 dominios (autocuidado, actividades domésticas, actividades físicas, actividades de ocio) donde se analiza la afectación de la disnea durante las actividades de la vida diaria de los pacientes con EPOC. Los valores más altos indican una mayor limitación en la realización de las actividades de la vida diaria ${ }^{26}$.

El cuestionario de salud EuroQol-5D (EQ$5 \mathrm{D})^{27}$ se empleó para la medición del estado percibido de salud. Está compuesta de dos secciones, una parte descriptiva y una escala visual analógica. La parte descriptiva dividida en 5 dimensiones (con 3 niveles cada una en función de la gravedad): movilidad, cuidado personal, actividades cotidia- 
nas, dolor/malestar, y ansiedad y depresión. La escala visual analógica evalúa el estado general de salud desde 0 (peor estado de salud imaginable) a 100 (mejor estado de salud imaginable).

\section{Análisis estadístico}

El análisis estadístico de los datos se realizó utilizando el software SPSS versión 20.0 para Windows. Las variables continuas fueron presentadas mediante la media \pm desviación estándar, en cambio, las variables categóricas, fueron presentadas en porcentajes. Para comprobar si las variables cuantitativas seguían una distribución normal se utiliza la prueba de Kolmogorov-Smirnow. En las variables cuantitativas se analizan las diferencias entre grupos mediante las pruebas $t$ de Student para diferencia de medias. El nivel de la significación estadística fue de $\mathrm{p} \leq 0,05$, al $95 \%$ de confianza.

La muestra fue dividida en dos grupos según la presencia de SL utilizándose la escala 25-question Geriatric Locomotive Function Scale (GFLS-25) ${ }^{28}$ en función de un punto de corte: el grupo $\mathrm{Pa}$ cientes EPOC sin SL (GFLS-25 < 16) y el grupo Paciente EPOC con SL (GFLS-25 $\geq 16$ ). Consta de 25 ítems referidos al dolor, las actividades de la vida diaria, la función social y al estado de salud mental del último mes. Se puntúa de 0 (no deterioro) a 4 (deterioro severo).

\section{Resultados}

Del total de 310 pacientes fueron incluidos finalmente 259, de los cuales fueron divididos en dos grupos (139 Pacientes EPOC con SL, 120 Pacientes EPOC sin SL). La distribución de los participantes se puede observar en la Figura 1.

Las características clínicas y sociodemográficas de los pacientes se muestran en la Tabla 1 .

La mayoría de los pacientes en ambo grupos son hombres $(76,4 \%$ pacientes EPOC con SL y $88,2 \%$ pacientes EPOC sin SL) y la edad media entre los pacientes no muestra diferencias significativas $(75,6 \pm 6,1$ pacientes EPOC con SL y $70,4 \pm 11,2$ pacientes EPOC $\sin$ SL). Se mostraron diferencias significativas entre ambos grupos en el número de comorbilidades, siendo mayor para el grupo con SL ( $p=0,036)$. En la función respiratoria valorada con $\mathrm{FEV}_{1}$ no se observan diferencias clínicamente significativas. Sin embargo, la disnea percibida presenta diferencias significativas entre grupos, siendo mayor en los pacientes EPOC con SL $(4,6 \pm 2,2, \mathrm{p}<0,001)$.

En cuanto al estado físico se mostraron diferencias significativas entre ambos grupos en la fuerza de miembro superior $(\mathrm{p}<0,001)$ y en los niveles de la actividad física $(\mathrm{p}=0,008)$, siendo los pacientes EPOC con SL los que presentan un deterioro en ambas variables. Sin embargo, la ca-

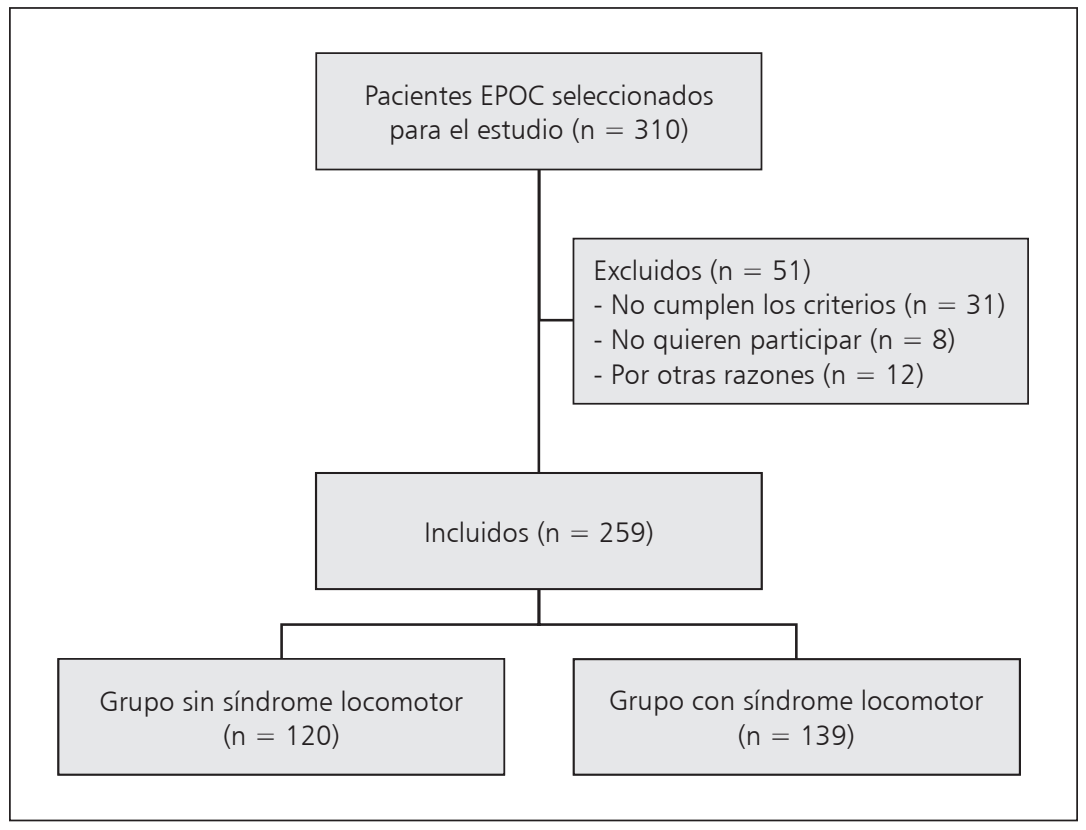

Figura 1. Diagrama de flujo de los participantes incluidos en el estudio. 
Tabla 1. Características clínicas de la muestra por grupo

\begin{tabular}{|c|c|c|c|}
\hline & $\begin{array}{l}\text { Pacientes EPOC con SL } \\
\qquad(n=139)\end{array}$ & $\begin{array}{l}\text { Pacientes EPOC sin SL } \\
\qquad(n=120)\end{array}$ & p-valor \\
\hline Edad (años) & $75,6 \pm 6,1$ & $70,4 \pm 11,2$ & 0,362 \\
\hline Sexo (\% hombres) & 76,4 & 88,2 & 0,864 \\
\hline $\mathrm{IMC}\left(\mathrm{kg} / \mathrm{m}^{2}\right)$ & $25,5 \pm 4,6$ & $22,9 \pm 6,1$ & 0,147 \\
\hline Índice de Charlson & $3,3 \pm 2,7$ & $2,7 \pm 1,8$ & $0,036^{*}$ \\
\hline $\mathrm{FEV}_{1} \%$ & $40,5 \pm 23,8$ & $49,4 \pm 29,9$ & 0,36 \\
\hline Borg Modificada & $4,6 \pm 2,2$ & $3,2 \pm 2,1$ & $p<0,001^{* *}$ \\
\hline \multicolumn{4}{|l|}{ Estado físico } \\
\hline Dinamometría MD & $228,4 \pm 78,4$ & $276,4 \pm 76,4$ & $p<0,001^{* *}$ \\
\hline $5 S T S$ & $25,7 \pm 8,9$ & $21,6 \pm 13,6$ & 0,068 \\
\hline MBPAQ & $3,7 \pm 3,4$ & $6,1 \pm 5,1$ & $0,008^{*}$ \\
\hline
\end{tabular}

Datos expresados en media \pm DT. *Entre grupos diferencias significativas $p<0,05$. **Entre grupos diferencias significativas $p \leq 0,001$. SL: Síndrome Locomotor; IMC: Índice de masa corporal; FEV1: Volumen espiratorio forzado en el primer segundo; MD; Miembro derecho; 5STS: Five Time-Sit-to-Stand Test; MBPAQ: Modified Baecke Physical Activity Questionnaire.

pacidad al ejercicio medida con 5STS no muestra diferencias significativas.

El estado funcional y la calidad de vida de los pacientes con EPOC se presentan en la Tabla 2.

El estado funcional relacionado con la disnea de los pacientes incluidos en el estudio mostró diferencias significativas en las variables autocui- dado y en el estado funcional total ( $\mathrm{p}<0,001)$. Además, se observó cambios significativos en los dominios de actividades domésticas, las actividades físicas y las actividades de ocio $(\mathrm{p}<0,05)$ indicando una disminución del estado funcional en los pacientes EPOC con SL frente a los pacientes EPOC sin SL.

Tabla 2. Diferencias en el estado funcional y calidad de vida de ambos grupos

\begin{tabular}{|c|c|c|c|}
\hline & $\begin{array}{l}\text { Pacientes EPOC con SL } \\
\qquad(n=139)\end{array}$ & $\begin{array}{l}\text { Pacientes EPOC sin SL } \\
(n=120)\end{array}$ & p-valor \\
\hline \multicolumn{4}{|l|}{ LCADL } \\
\hline Autocuidado & $11,33 \pm 4,58$ & $6,98 \pm 3,44$ & $p<0,001^{* *}$ \\
\hline Actividades domésticas & $7,90 \pm 10,93$ & $5,02 \pm 7,82$ & 0,115 \\
\hline Actividades físicas & $6,31 \pm 2,36$ & $5,3 \pm 2,18$ & $0,015^{*}$ \\
\hline Actividades de ocio & $6,78 \pm 2,73$ & $5,49 \pm 2,92$ & $0,01^{*}$ \\
\hline Total & $32,27 \pm 13,64$ & $22,79 \pm 11,21$ & $p<0,001^{* *}$ \\
\hline \multicolumn{4}{|l|}{ EQ-5D } \\
\hline Movilidad & $1,853 \pm 0,432$ & $1,225 \pm 0,372$ & $p<0,001^{* *}$ \\
\hline Cuidado personal & $1,899 \pm 0,692$ & $1,275 \pm 0,419$ & $p<0,001^{* *}$ \\
\hline Actividades cotidianas & $1,871 \pm 0,658$ & $1,313 \pm 0,511$ & $p<0,001^{* *}$ \\
\hline Dolor & $1,532 \pm 0,549$ & $1,225 \pm 0,404$ & $p<0,001^{* *}$ \\
\hline Ansiedad y Depresión & $1,799 \pm 0,598$ & $1,313 \pm 0,459$ & $p<0,001^{* *}$ \\
\hline EVA & $50,453 \pm 17,194$ & $64,025 \pm 18,890$ & $p<0,001^{* *}$ \\
\hline
\end{tabular}

Datos expresados en media \pm DT. * Entre grupos diferencias significativas $p<0,05 .{ }^{* *}$ Entre grupos diferencias significativas $\mathrm{p} \leq$ 0,001. SL: Síndrome Locomotor; LCADL: London Chest Activity of Daily Living scale; EQ-5D: EuroQol-5D; EVA: Escala Visual Analógica. 
En cuanto a la calidad de vida se encontraron diferencias significativas entre ambos grupos en la movilidad, el cuidado personal, las actividades cotidianas, el dolor, la ansiedad y depresión, y la EVA $(\mathrm{p}<0,001)$, siendo el de pacientes EPOC con SL el que mostraba menos calidad de vida.

\section{Discusión}

El objetivo de este estudio fue evaluar la prevalencia del SL y valorar la repercusión que tiene en la funcionalidad y en la calidad de vida de los pacientes con EPOC. Previos estudios en pacientes con EPOC $^{29,30}$ han empleado un tamaño de muestra similar a nuestro estudio para detectar diferencias mínimas significativas en las variables a estudio.

Nuestros resultados han mostrado que existe diferencias significativas en el estado físico de los pacientes. Se observaron cambios significativos en la dinamometría de miembro derecho $(\mathrm{p}<0,001)$ $y$ en el nivel de actividad física $(p=0,008)$, mostrando una disminución del estado físico en los pacientes EPOC con SL. Sin embargo, no se observaron diferencias significativas en la capacidad al ejercicio. La actividad física, actividades de ocio, autocuidado y estado funcional relacionado con la disnea mostró un deterioro significativo para los pacientes EPOC con SL. Además, se observó una disminución de la calidad de vida en aquellos pacientes EPOC que presentaban SL $(\mathrm{p}<0,001)$.

Según diversos estudios ${ }^{31,32}$ hay un incremento de la EPOC en la población masculina a partir de los 40 años. Además, Cheung et al. ${ }^{33}$ observó que el 90\% del aparato locomotor comienza a deteriorarse a partir de los 55 años. Por ello, nuestros resultados junto con los de otros estudios ${ }^{34,35}$ han observado en hombres mayores de 45 años $(82,3 \%)$ una alta prevalencia de deterioro en el estado funcional y físico derivado del SL.

El paciente con EPOC sufre un deterioro progresivo de la enfermedad donde principalmente se observa un aumento de la disnea que dificulta la realización de las actividades de la vida diaria ${ }^{36}$. El perfil clínico propio de la enfermedad crónica junto con los factores asociados a SL impacta de manera negativa sobre la fuerza, velocidad de la marcha, y consumo máximo de oxígeno ${ }^{2}$. Por ello, aquellos pacientes que padecen EPOC y SL refieren un deterioro significativo en el estado funcional relacionado con la disnea y en el estado físico general mayor que aquellos que no padecen SL.

Adicionalmente, existe una disfunción junto con el progreso de la enfermedad en las actividades de la vida diaria incluyendo actividades básicas necesarias para el autocuidado y actividades instrumentales $^{37}$. Nuestro estudio ha demostrado que los pacientes con EPOC sufren un deterioro significativo cuando presentan SL en las actividades de la vida diaria relacionadas con la disnea. A medida que se afectan estas actividades se produce un aumento la dependencia del paciente que deriva en la necesidad de ayuda externa a través del cuidador ${ }^{38}$.

Waltson J et al. ${ }^{39}$ observó que el SL ocasiona un deterioro fisiológico. Esto implica alteraciones inmunológicas, alteraciones endocrinas y la reducción del tejido musculo-esquelético que disminuyen la capacidad de afrontar efectos adversos producidos por la enfermedad. El curso de la EPOC está caracterizado por períodos de exacerbación donde el paciente sufre un deterioro del estado clínico ${ }^{40}$. Nuestro estudio ha demostrado que las alteraciones fisiológicas derivadas del SL junto con las características clínicas del EPOC producen un deterioro mayor del estado funcional y de la calidad de vida, lo cual se podría ver incrementado en los periodos de exacerbación.

Actualmente, la EPOC es problema de salud pública que requiere de acciones preventivas y de promoción de salud inmediatas debido a la alta prevalencia de la enfermedad ${ }^{41}$. Los resultados de nuestro estudio han mostrado un deterioro funcional y de calidad de vida significativamente mayor en los pacientes EPOC con SL. Por ello, en esta población las actividades relacionadas con la salud publica deben ir enfocadas a la instauración de intervenciones educativas que promuevan cambios de comportamiento y estilo de vida a largo plazo, como rehabilitación física y pulmonar ${ }^{42}$.

Según el estudio de Kataoka et al. ${ }^{14}$ realizado sobre pacientes con síndrome obstructivo de la apnea del sueño se observó una disminución de la calidad de vida en los pacientes sin SL. Además, se mostró que la calidad de vida en estos pacientes era independiente del SL. Sin embargo, nuestro estudio mostró diferencias significativas entre ambos grupos ( $\mathrm{p}<0,001)$, indicando un deterioro en la calidad de vida de los pacientes con EPOC y SL.

Diversas limitaciones fueron encontradas en nuestro estudio. En primer lugar, el curso de la 
EPOC es caracterizado por un empeoramiento clínico y sintomatológico que no ha sido evaluado y podría dar mayor validez al estudio. En segundo lugar, el diseño observacional transversal realizado en este estudio no permite evaluar el deterioro funcional y de la calidad de vida durante la enfermedad.

El SL en pacientes con EPOC produce un cambio significativo en el estado físico que produce un deterioro del estado funcional repercutiendo en las actividades de la vida diaria disminuyendo la calidad de vida estos pacientes. Por lo tanto, se necesitan más proyectos que estudien esta materia para determinar cómo influye el SL durante el progreso del EPOC.

\section{Referencias}

1. Ikemoto T, Arai YC. Locomotive syndrome: clinical perspectives. Clin Interv Aging. 2018; 30 (13): 819-27.

2. Nakamura K, Ogata T. Locomotive syndrome: definition and management. Clin Rev Bone Miner Metab. 2016; 14 (2): 56-67.

3. Theou O, Cann L, Blodgett J, Wallace LM, Brothers TD, Rockwood K. Modifications to the frailty phenotype criteria: Systematic review of the current literature and investigation of 262 frailty phenotypes in the Survey of Health, Ageing, and Retirement in Europe. Ageing Res Rev. 2015; 21: 78-94.

4. Nakamura M, Kobashi Y, Hashizume H, Oka H, Kono $\mathrm{R}$, Nomura $\mathrm{S}$, et al. Locomotive syndrome is associated with body composition and cardiometabolic disorders in elderly Japanese women. BMC Geriatric. 2016; 16 (1): 166.

5. Mercado N, Ito K, Barnes PJ. Accelerated ageing of the lung in COPD: new concepts. Thorax. 2015; 70 (5): 4829.

6. Torres-Sánchez I, Cabrera-Martos I, Díaz-Pelegrina A, Valenza-Demet G, Moreno-Ramírez MP, Valenza MC. Physical and Functional Impairment During and After Hospitalization in Subjects With Severe COPD Exacerbation. Respir Care. 2017; 62 (2): 209-14.

7. Peces-Barba G, Barberà JA, Agustí À, Casanova C, Casas A, Izquierdo JL, et al. Joint guidelines of the Spanish Society of Pulmonology and Thoracic Surgery (SEPAR) and the Latin American Thoracic Society (ALAT) on the diagnosis and management of chronic obstructive pulmonary disease. Arch Bronconeumol. 2008; 44 (5): 271-81.

8. Celli BR, MacNee W, ATS/ERS Task Force. Standards for the diagnosis and treatment of patients with COPD: a summary of the ATS/ERS position paper. Eur Respir J. 2004; 23 (6): 932-46.

9. Alonso JI, Paredes CM. Enfermedad pulmonar obstructiva crónica (EPOC). Medicine-Programa de Formación Médica Continuada Acreditado 2018; 12 (63): 3699-709.

10. Cielen N, Maes K, Gayan-Ramírez G. Musculoskeletal disorders in chronic obstructive pulmonary disease. Biomed Res Int. 2014; 2014: 965764.

11. Freemont A, Hoyland J. Morphology, mechanisms and pathology of musculoskeletal ageing. The Journal of Pathology: A Journal of the Pathological Society of Great Britain and Ireland. 2007; 211 (2): 252-9.

12. Matsumoto H, Hagino H, Wada T, Kobayashi E. Locomotive syndrome presents a risk for falls and fractures in the elderly Japanese population. Osteoporos Sarcopenia 2016; 2 (3): 156-63.

13. Nakamura M, Hashizume $H$, Nomura S, Kono R, Utsunomiya $\mathrm{H}$. The relationship between locomotive syndrome and depression in community-dwelling elderly people. Current gerontology and geriatrics research 2017; 2017: 4104802.

14. Kataoka H, Miyatake N, Ichikawa H, Arakawa Y, Mori Y. Relationship of locomotive syndrome with health-related quality of life among patients with obstructive sleep apnea syndrome. J Phys Ther Sci. 2017; 29 (7): 1129-33.

15. Pauwels RA, Buist AS, Calverley PM, Jenkins CR, Hurd SS. Global strategy for the diagnosis, management, and prevention of chronic obstructive pulmonary disease: NHLBI/WHO Global Initiative for Chronic Obstructive Lung Disease (GOLD) Workshop summary. Am J Respir Crit Care Med. 2001; 163 (5): 1256-76.

16. Charlson ME, Pompei P, Ales KL, MacKenzie CR. A new method of classifying prognostic comorbidity in longitudinal studies: development and validation. J Chronic Dis. 1987; 40 (5): 373-83.

17. García Río F, Calle M, Burgos F, Casan P, del Campo F, Galdiz JB, et al. Espirometría. Arch Bronconeumol. 2013; 49 (9): 388-401.

18. Clarà PC. La espirometría en la práctica médica. Archivos de medicina del deporte. Revista de la Federación Española de Medicina del Deporte y de la Confederación Iberoamericana de Medicina del Deporte 2003; 96: 347.

19. Borg GA. Psychophysical bases of perceived exertion. Med Sci Sports Exerc. 1982; 14 (5): 377-81.

20. Vallejo JMB, Rosique DF, Ros EH, González-Moro IM. Fuerza máxima y resistencia muscular de agarre manual en regatistas de vela ligera de la clase Tornado. Apunts. Medicina de l'Esport 2007; 42 (156): 161-8. 
21. Paltamaa J, West H, Sarasoja T, Wikström J, Mälkiä E. Reliability of physical functioning measures in ambulatory subjects with MS. Physiotherapy Research International 2005; 10 (2): 93-109.

22. Mateo Lázaro M, Berisa Losantos F, Plaza Bayo A. Nuevas tablas de fuerza de la mano para población adulta de Teruel. Nutr Hosp. 2008; 23 (1): 35-40.

23. Hortobágyi T, Mizelle C, Beam S, DeVita P. Old adults perform activities of daily living near their maximal capabilities. J Gerontol A Biol Sci Med Sci. 2003; 58 (5): M453-M460.

24. Wretenberg P, Arborelius UP. Power and work produced in different leg muscle groups when rising from a chair. Eur J Appl Physiol Occup Physiol. 1994; 68 (5): 413-7.

25. McCarthy EK, Horvat MA, Holtsberg PA, Wisenbaker JM. Repeated chair stands as a measure of lower limb strength in sexagenarian women. Journals of Gerontology: Medical Sciences. 2004; 59A: 1207-12.

26. Garrod R, Bestall J, Paul E, Wedzicha J, Jones P. Development and validation of a standardized measure of activity of daily living in patients with severe COPD: the London Chest Activity of Daily Living scale (LCADL). Respir Med. 2000; 94 (6): 589-96.

27. Group TE. EuroQol-a new facility for the measurement of health-related quality of life. Health Policy 1990; 16 (3): 199-208.

28. Seichi A, Hoshino Y, Doi T, Akai M, Tobimatsu Y, Iwaya T. Development of a screening tool for risk of locomotive syndrome in the elderly: the 25-question Geriatric Locomotive Function Scale. Journal of Orthopaedic Science 2012; 17 (2): 163-72.

29. Grolimund E, Kutz A, Marlowe RJ, Vögeli A, Alan M, Christ-Crain $\mathrm{M}$, et al. Long-term prognosis in COPD exacerbation: role of biomarkers, clinical variables and exacerbation type. COPD 2015; 12 (3): 300-10.

30. Johansson Strandkvist V, Backman H, Andersson M, Larsson A, Stridsman C, Lindberg A. Hand grip strength is associated with fatigue in COPD. Int J Chron Obstruct Pulmon Dis. 2017; 11: 2527-34.

31. Tinkelman DG, Price DB, Nordyke RJ, Halbert RJ. Misdiagnosis of COPD and asthma in primary care patients 40 years of age and over. Journal of Asthma. 2006; 43 (1): 75-80.

32. Fletcher MJ, Upton J, Taylor-Fishwick J, Buist SA, Jenkins C, Hutton J, et al. COPD uncovered: an inter- national survey on the impact of chronic obstructive pulmonary disease [COPD] on a working age population. BMC public health. 2011; 11 (1): 612.

33. Cheung KM, Karppinen J, Chan D, Ho DW, Song Y, Sham P, et al. Prevalence and pattern of lumbar magnetic resonance imaging changes in a population study of one thousand forty-three individuals. Spine 2009; 34 (9): 934-40.

34. Miyawaki T, Kumamoto K, Shimoda K, Tozato F, Iwaya T. Relationship among motor function, ADL disability, and psychological concerns in elderly people with locomotive disorders. Journal of Orthopaedic Science 2017; 22 (2): 339-44.

35. Cortopassi F, Gurung P, Pinto-Plata V. Chronic Obstructive Pulmonary Disease in Elderly Patients. Clin Geriatr Med. 2017; 33 (4): 539-52.

36. Miravitlles M, Soriano JB, Garcia-Rio F, Munoz L, Duran-Tauleria E, Sanchez G, et al. Prevalence of COPD in Spain: impact of undiagnosed COPD on quality of life and daily life activities. Thorax 2009; 64 (10): 8638.

37. Braido F, Baiardini I, Scichilone N, Sorino C, Di Marco F, Corsico A, et al. Disability in moderate chronic obstructive pulmonary disease: Prevalence, burden and assessment - results from a real-life study. Respiration 2015; 89 (2): 100-6.

38. Weinreich UM, Kaptajn RJ, Helle T. Need of help for activities of daily living in patients with mild to severe COPD: Who helps, with what and how much? European Respiratory Journal. 2020; 56: 955.

39. Walston J, Hadley EC, Ferrucci L, Guralnik JM, Newman AB, Studenski SA, et al. Research agenda for frailty in older adults: toward a better understanding of physiology and etiology: summary from the American Geriatrics Society/National Institute on Aging Research Conference on Frailty in Older Adults. J Am Geriatr Soc. 2006; 54 (6): 991-1001.

40. Aaron SD, Donaldson GC, Whitmore GA, Hurst JR, Ramsay T, Wedzicha JA. Time course and pattern of COPD exacerbation onset. Thorax. 2012; 67 (3): 238-43.

41. Kiley JP, Gibbons GH. COPD National Action Plan: Addressing a Public Health Need Together. Chest. 2017; 152 (4): 698-9.

42. Marillier M, Bernard AC, Vergès S, Neder JA. Locomotor Muscles in COPD: The Rationale for Rehabilitative Exercise Training. Front Physiol. 2020; 10: 1590. 